\title{
Comportamiento pernicioso: el efecto de las consecuencias en la elección entre perjudicar/no perjudicar a un compañero*
}

Pernicious Behavior: the Effect of the Consequences in the Choice between to Harm/do not Harm a Classmate

\author{
Claudio Carpio \\ Universidad Nacional Autónoma de México, México \\ Héctor Silva \\ Universidad Nacional Autónoma de México, México \\ HÉctor Garduño \\ Universidad Nacional Autónoma de México, México \\ LindA PACHECO \\ Universidad Nacional Autónoma de México, México \\ Raúl RodRíGuez \\ Universidad Nacional Autónoma de México, México \\ Maricela Chaparro \\ Universidad Nacional Autónoma de México, México \\ Jamillet Carranza \\ Universidad Nacional Autónoma de México, México \\ Germán Morales ${ }^{a}$ \\ Universidad Nacional Autónoma de México, México \\ ORCID: http://orcid.org/0000-0002-7336-5743
}

\footnotetext{
a Autor de correspondencia. Correo electrónico: gmoralesc@unam.mx
}

Para citar este artículo: Carpio, C., Silva, H., Garduño, H., Pacheco, L., Rodríguez, R., Chaparro, M., Carranza, J., \& Morales, G. (2018). El comportamiento pernicioso: el efecto de las consecuencias en la elección entre perjudicar/no perjudicar a un compañero. Universitas Psychologica, 17(3), 1-12. https://doi.org/10.11144/Javeriana.upsy 17-3.cpec

\section{RESUMEN}

La presente investigación, se realizó con base en dos supuestos interconductuales: 1) el comportamiento es susceptible de modularse por factores históricos y/o situacionales; 2) el comportamiento pernicioso puede regularse por factores situacionales como las consecuencias. El objetivo fue evaluar el efecto de las consecuencias (positivas y negativas) sobre la elección entre perjudicar o no a un compañero durante la solución de una tarea. Participaron 35 estudiantes universitarios distribuidos aleatoriamente en cinco grupos. La tarea experimental consistió en la solución de operaciones aritméticas proporcionalmente puntuadas (1 a 5 puntos). El principal resultado fue que las consecuencias negativas por no perjudicar modulan mayoritariamente la ocurrencia de comportamiento pernicioso. Los resultados se discuten en términos del papel regulador de las consecuencias (en relación con el comportamiento pernicioso) en tanto factor situacional.

\section{Palabras clave}

comportamiento social; comportamiento moral; perjudicar; ayudar; consecuencias.

\section{ABSTRACT}

This research was based on two main interbehavioral suppositions: 1) Behavior is likely modulated by historical and/or situational factors; 
2) Pernicious behavior can be regulated by situational factors such as the consequences. The objective was to evaluate the effect of the consequences (positives and negatives) on the choice between to harm or do not harm a classmate while solving a task. Thirty-five college students were randomly assigned into one of five experimental groups. The task consisted in solving arithmetic operations proportionally scored from 1 to 5 points. The results are discussed in terms of the regulatory role of the consequences (in relation to pernicious behavior) as a situational factor. The main result was that the negative consequences by the choice of do not harm the partner mainly modulated the occurrence of pernicious behavior.

Keywords

social behavior; moral behavior; harm; help; consequences.

Desde el establecimiento de las primeras formaciones gregarias, el ser humano ha destacado con respecto a los organismos subhumanos en la medida en que se comporta de acuerdo con criterios convencionales y, por consiguiente, los individuos miembros de un grupo social en particular se ajustan a prácticas compartidas en aras de pertenecer a éste (Ribes-Iñesta, Rangel, \& LópezValadez, 2008). Cada grupo social define, establece, da forma y sentido a un conjunto de ideas, creencias y valoraciones sobre el significado que tiene pertenecer al mismo, delimitando comportamientos, características e incluso pensamientos y emociones que son adecuados para cada ser humano, con base a esta red de ideas consensuadas (RochaSánchez \& Díaz-Loving, 2005). De acuerdo con estos criterios compartidos en la forma de sistemas reactivos convencionales, se estipulan reglas, leyes, normas, preceptos, estatutos e instrucciones de convivencia que procuran el bienestar individual y colectivo. En esta línea de ideas, se prescriben estándares de comportamiento para los individuos de una sociedad determinada, teniendo lugar lo que ordinariamente se denomina como: moralidad.

La moral junto con la dimensión legal de las formaciones sociales, han dispuesto al ser humano a explicitar la forma esperada de actuar y, por lo tanto, conductas tales como matar, robar, mentir, etcétera, que afectan de manera negativa a otros individuos, están prohibidas, sancionadas por las autoridades sociales y son reprobadas por los miembros del grupo social.

En la actualidad el aumento en la comisión y gravedad de este tipo de conductas ha propiciado que sociólogos, pedagogos y psicólogos sobre todo, realicen investigaciones con el objeto de caracterizarlas u modificarlas, planteándose hegemónicamente el siguiente cuestionamiento: ¿Por qué los individuos perjudican a otros? Esta interrogante ha suscitado el acercamiento e intervención a diversos fenómenos en los que la constante es perjudicar a otro, por ejemplo, el llamado 'bullying' en ámbitos educativos (Van der Werf, 2014), el 'mobbing' en ámbitos laborales (Lima \& Souza, 2015), entre otros.

En la sociedad mexicana se presentan estos fenómenos que, sin duda, trastocan de manera significativa el comportamiento individual. Es decir, la conducta de los miembros del grupo social está afectada y regulada por factores de naturaleza socio-cultural, así como por otros factores adicionales. Autores como Rogoff (1993) y Lave \& Wenger (1991) sostienen que el entorno cultural determina el comportamiento individual. Distintas investigaciones (Guerrero, 2010) sugieren que algunos factores de carácter biológico desempeñan un papel crítico en relación con la ocurrencia de conductas perjudiciales. Sin embargo, Carpio et al. (2008a) y Carpio et al. (2008b), afirman que los factores culturales y biológicos son variables que probabilizan, mas no determinan el comportamiento individual.

Una serie de estudios realizados en la década de los sesenta (Milgram, 2005) ponen en evidencia el papel de factores situacionales, en dichos estudios se utilizó un aparato generador de choques eléctricos que los participantes controlaban, es decir, estos suponían que administraban choques eléctricos a otro participante (experimentador coludido) en una situación de aprendizaje. Los choques eléctricos se simulaban (los participantes desconocían este respecto). Participaron 40 hombres cuyas edades oscilaron entre $20 \mathrm{y}$ 50 años, el objetivo consistió en evaluar el comportamiento de los participantes ante una figura de autoridad, cada participante fue 
colocado en una situación en la que se le indico que perjudicara a otro. La mayoría (65\%) mostraron una proclividad a perjudicar si se les demanda hacerlo. Ulteriores experimentos constataron que las mujeres se comportan de manera similar respecto a los hombres (Milgram, 2005). Burger (2009) replicó el experimento, hallando que el $77 \%$ de los participantes opta perjudicar en tanto se les establece hacerlo.

En este sentido, las conductas realizadas con la pretensión de dañar a un individuo se denominan, de acuerdo con el lenguaje ordinario: actos perjuiciosos, perjudiciales o que se inclinan a perjudicar. Con propósitos analíticos se le llamará comportamiento pernicioso, a este tipo de conducta y se conceptualizará como aquellas interacciones, en las cuales, se identifica una afectación negativa del campo contingencial de un individuo en específico. Esto constituye una alteración negativa de las relaciones de interdependencia respecto de las cuales se comporta un individuo. El comportamiento pernicioso, se caracteriza con base en los supuestos teóricos explicitados por Kantor y Smith (1975) y la taxonomía de la organización funcional de la conducta formulada por Ribes y López (1985). Estos autores proponen a la interconducta como objeto de conocimiento de la disciplina psicológica, es decir, la interacción entre un organismo individuo y un aspecto específico del medio ambiente; esta interacción se configura históricamente y se encuentra modulada por factores disposicionales (organísmicos, situacionales, entre otros).

Ribes y López (1985) aseveran que las interacciones psicológicas difieren en términos de la organización funcional de los elementos involucrados. Con esta base, se establecen funciones estímulo-respuesta, lo cual indica que se identifica una estructuración funcional entre los eventos de estímulo y respuesta; las funciones estímulo-respuesta son indisolubles.

En relación con la caracterización del comportamiento pernicioso, se consideran los siguientes elementos participantes: a) Al menos dos individuos; b) Un criterio a satisfacer mediante la realización de actividades; c) Una situación problema; d) La elección de uno | Universitas Psychologica | V. I7 | No. 3 | 2018 | de los individuos entre realizar actividades en aras de afectar negativamente el campo contingencial del otro individuo, posponiendo la satisfacción del criterio propio, o bien, negarse a afectar de manera negativa el campo contingencial del otro individuo $y$, por ende, satisfacer el criterio propio; e) Factores disposicionales: elementos que condicionan probabilísticamente una interacción. Por ejemplo, consecuencias, historia referencial, historia situacional, nivel competencial, restricción temporal, magnitud de las consecuencias, conocimiento de la actividad ajena, entre otros.

En los estudios de Carpio et al. (2008a, 2008b) se ha documentado que el comportamiento solidario está modulado por factores situacionales como las consecuencias, observándose un efecto diferencial en correspondencia con dichas consecuencias. Esto implica que si un individuo ayuda a otro a cumplir con su tarea en función de aquello que pierde, más que de aquello que gana ayudando a otro. Este resultado se ha replicado también en condiciones en las que se ofrece un discurso sobre lo 'bueno' o 'malo' que es ayudar, jugando un papel más relevante, las consecuencias por ayudar o no ayudar que los segmentos lingüísticos sobre ayudar/no ayudar. En lo que toca a las consecuencias, los resultados permiten sostener que las consecuencias situacionales inmediatas desempeñan un papel modulador en la elección entre ayudar o no ayudar a un compañero. En particular, la presentación de consecuencias negativas ante la elección no ayudar favorece la ocurrencia de comportamiento solidario. Sin embargo, se observó una asimetría en relación con la calidad del desempeño, esto evidenció que los participantes solucionaron operaciones aritméticas correctamente durante la tarea propia, disminuyendo la cantidad de operaciones solucionadas de manera correcta en lo concerniente a la tarea ajena. En cambio, las consecuencias positivas por ayudar, positivas por no ayudar, negativas por ayudar y la ausencia de consecuencias, suponen una proclividad por no ayudar.

En resumen, el comportamiento de ayudar o no ayudar, depende de factores situacionales, 
como las consecuencias y por ende, es susceptible de modificarse. Considerando que el comportamiento de un individuo está regulado por factores que se han incorporado en su historia y por factores situacionales, como las consecuencias, más que por supuestas propiedades intrínsecas en los individuos, entonces, es posible pensar que el comportamiento pernicioso no es un tipo de fenómeno cualitativamente diferente al comportamiento de ayudar, sino una variación paramétrica que adopta una forma particular. Por ende, es pertinente esperar que las consecuencias jueguen un papel modulador en la emergencia del comportamiento pernicioso. Para explorar empíricamente esta posibilidad, se planteó como objetivo de la presente investigación, evaluar los efectos de dos tipos de consecuencias: positivas y negativas, sobre la elección entre perjudicar/ no perjudicar a un compañero en una tarea aritmética. Con la finalidad de contrastar los datos obtenidos experimentalmente entre el comportamiento de ayudar y el comportamiento pernicioso se empleó una tarea similar.

\section{Método}

\section{Participantes}

Participaron voluntariamente 35 estudiantes (18 mujeres y 17 hombres) de la Facultad de Estudios Superiores Iztacala (F.E.S.I.) de la Universidad Nacional Autónoma de México (U.N.A.M.), cuyas edades oscilaron entre 18 y 25 años.

\section{Aparatos e instrumentos}

Se utilizaron siete computadoras de escritorio con un sistema operativo Microsoft XP Profesional y la plataforma de programación Visual Basic 6.0. Se diseñó un programa de cómputo con esta plataforma, basándose en el programa empleado para el análisis experimental relativo al comportamiento solidario -situación de elección ayudar/no ayudar- (Cantoran, 2007). Así entonces, se configuró la tarea de evaluación experimental. Se ocuparon hojas blancas tamaño carta y lápices para la solución de las operaciones aritméticas.

\section{Situación experimental}

Las sesiones experimentales se llevaron a cabo en el Laboratorio de Análisis de Procesos Psicológicos Superiores, localizado en la Clínica Universitaria de Salud Integral (C.U.S.I.) de la F.E.S.I. Se dispuso de siete Estaciones de Evaluación Experimental (E.E.E.), con un equipo de cómputo (monitor, teclado, ratón y C.P.U.) cada una. Las E.E.E. restringen la influencia de variables extrañas.

\section{Procedimiento}

Los participantes, fueron asignados aleatoriamente a uno de cinco grupos (cuatro grupos experimentales y uno control), conformados por siete participantes cada uno:

1) Consecuencias Positivas por Perjudicar (CPP); 2) Consecuencias negativas por perjudicar (CNP); 3) Consecuencias positivas por no perjudicar (CPNP); 4) Consecuencias negativas por no perjudicar (CNNP); 5) Sin consecuencias (SC). Las consecuencias positivas equivalían a la obtención de tres puntos, en tanto las consecuencias negativas equivalían a la remoción de tres puntos.

Una vez asignados los participantes a los cinco grupos, se les colocó en las E.E.E. de manera independiente. Es decir, uno a uno, se les indicó tomar asiento frente a una mesa, en la cual, fue dispuesto un monitor, teclado y ratón. Posteriormente, se dio inicio a la tarea experimental presentándose las siguientes instrucciones en la pantalla del monitor:

Tu tarea consiste en realizar operaciones aritméticas básicas (sumas, restas, multiplicaciones y divisiones) en aras de obtener 60 puntos en 30 minutos, tiempo límite. Podrás seleccionar el tipo de operación aritmética básica que desees solucionar (las sumas y las restas solucionadas correctamente equivalen a 1 punto, las multiplicaciones

| Universitas Psychologica | V. I7 | No.3 | 2018 | 
solucionadas correctamente equivalen a 3 puntos y, por último, las divisiones solucionadas correctamente equivalen a 5 puntos). Para realizar las operaciones aritméticas básicas utiliza las hojas blancas tamaño carta y el lápiz colocados a un costado del teclado. En tanto realices los cálculos necesarios para obtener el resultado correcto, ingresa éste en el botón correspondiente; coloca el cursor sobre el botón 'confirmar' y oprime la tecla derecha del ratón. Recuerda, si acumulas 60 puntos antes que tus compañeros entonces obtendrás un premio. iSuerte! Presiona el botón 'comenzar'.

Cuando el participante presionaba el botón 'comenzar', transcurrían diez segundos y se habilitaba un botón con la palabra 'continuar', al oprimirlo se presentaba otro panel, en el cual, aparecía una ventana para registrarse, a su vez, un conjunto de avatares. Los participantes seleccionaban uno; el avatar representaba a los participantes durante la tarea experimental.

Una vez seleccionado un avatar, se presentaba el siguiente panel, en el cual, aparecía una ventana con las palabras 'Tu tarea' (en la parte superior izquierda del panel). Igualmente se mostraba un contador con objeto de indicar la puntuación acumulada respecto de los 60 puntos requeridos; otro contador señalaba los puntajes de los otros participantes tres compañeros virtuales, los participantes desconocían este asunto. Los puntajes de éstos variaban respecto a la puntuación del participante en cuestión sin excepción, uno de los compañeros virtuales ostentaba un puntaje superior, otro un puntaje inferior y, finalmente, otro un puntaje próximo o idéntico respecto del participante en cuestión-. Por último, aparecía un recuadro para seleccionar el tipo de operación aritmética básica. Cuando el participante seleccionaba un tipo de operación aritmética, se mostraba (en la parte inferior del panel) una operación en correspondencia con la seleccionada anteriormente $y$, a su vez, un recuadro dispuesto para ingresar el resultado. Se utilizaba el teclado y el ratón para ejecutar esta acción. Si el resultado era correcto entonces se presentaba durante cinco segundos un mensaje con la expresión 'Tu resultado es correcto' y, por tanto, incrementaba la puntuación propia en función de la operación aritmética solucionada. En cambio, si el resultado era incorrecto aparecía durante cinco segundos un mensaje con la expresión 'Tu resultado es incorrecto' y se habilitaba nuevamente el recuadro referente a la selección de un tipo de operación aritmética en particular; evidentemente la puntuación propia se mantenía sin aumentar.

En cuanto el participante acumulaba veinte puntos, se presentaba un recuadro con el mensaje '¿Deseas realizar operaciones en aras de disminuir la puntuación de uno de tus compañeros?'. A la par, se mostraban dos botones con distintas opciones; uno con la frase 'Deseo realizar operaciones en aras de disminuir la puntuación de uno de los otros participantes' y otro botón con la frase 'Prefiero continuar con mi tarea' (ver Figura 1).

\section{Figura 1}

Elección entre perjudicar/no perjudicar a un compañero virtual

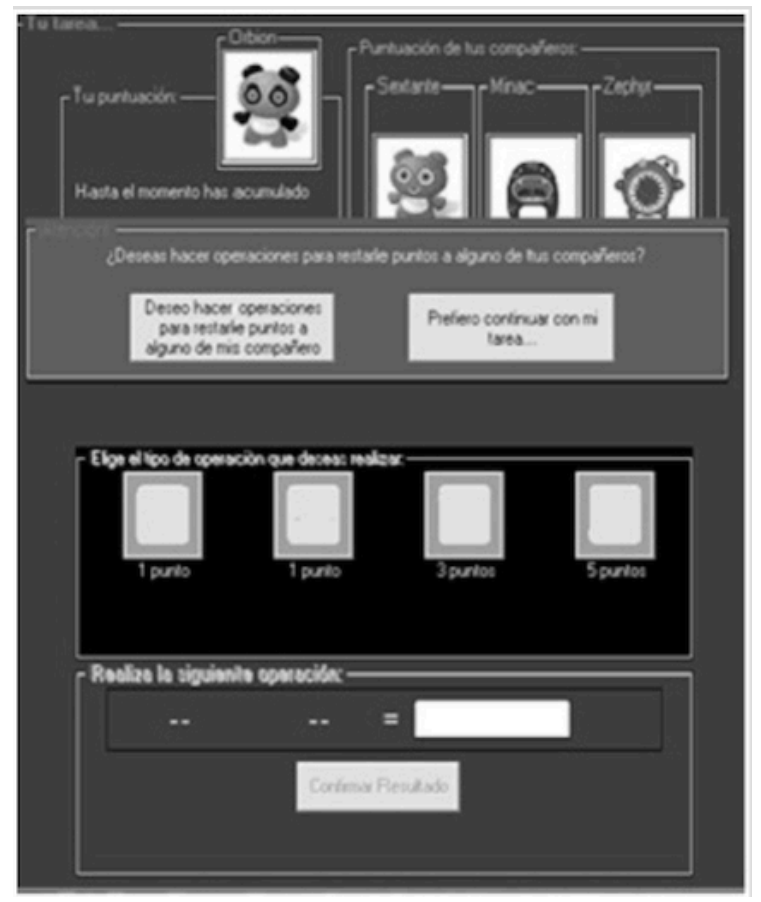

Si se oprimía el botón 'Prefiero continuar con mi tarea', se mantenía habilitado el panel correspondiente a la tarea propia. Por el contrario, si se presionaba el botón 'Deseo realizar operaciones en aras de disminuir la puntuación de uno de los otros participantes' se 
inhabilitaba el panel relativo a la tarea propia y se presentaba un recuadro con la pregunta 'iA cuál de tus compañeros deseas disminuirle puntos?'. Enseguida de la pregunta anterior (es decir, en la parte inferior) aparecían los avatares de los compañeros virtuales en conjunción con la puntuación de cada uno (ver Figura 2). El participante únicamente podía seleccionar a uno de los compañeros. Ulteriormente, se presentaba un panel con distinto color, en el cual, se mostraba la frase 'Tarea de tu compañero'. Este panel se operaba de manera idéntica con respecto al panel relativo a la tarea propia, con una excepción, que consistía en presentar el ícono del avatar del compañero virtual perjudicado en combinación con la puntuación acumulada de éste.

\section{Figura 2}

Selección de uno de los compañeros virtuales con la finalidad de disminuir su puntuación

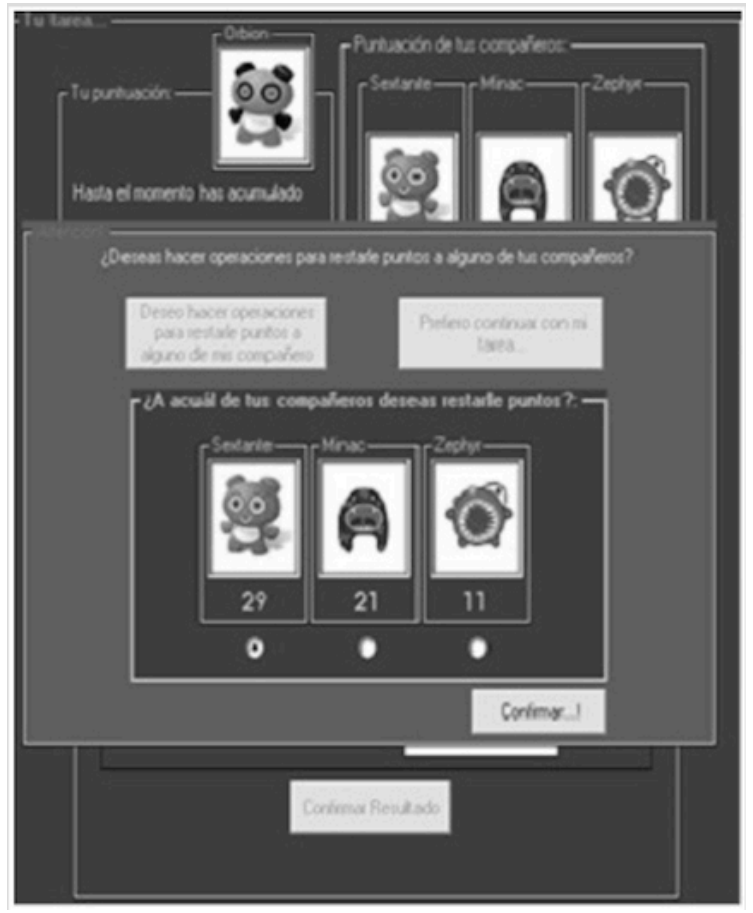

Posteriormente, el participante seleccionaba una operación aritmética básica con propósito de perjudicar al compañero virtual previamente elegido. Realizados los cálculos pertinentes y habiendo ingresado el resultado, se presentaba un mensaje notificador, indicando si el resultado era correcto o incorrecto. Independientemente del resultado (correcto o incorrecto), se disminuía la puntuación acumulada del compañero virtual en cuestión (de conformidad con la operación aritmética solucionada). Luego entonces, aparecía el siguiente mensaje 'Le has disminuido puntos a un compañero' en conjunción con un botón, en el cual, se mostraba el siguiente mensaje 'Presiona para continuar con tu tarea'; al oprimirlo se habilitaba el panel correspondiente a la tarea propia.

En relación con el grupo experimental CPP, si el participante oprimía el botón 'Prefiero continuar con mi tarea', se presentaba durante tres segundos (en el centro del panel) el mensaje 'iPor haber perjudicado a un compañero has obtenido 3 puntos!'. La puntuación acumulada incrementaba tres puntos y, en tanto se ejecutaba la sumatoria, el contador sobresalía debido a que se presentaba con una luz intermitente.

En cuanto al grupo experimental CNP, si el participante oprimía el botón 'Prefiero continuar con mi tarea' se presentaba durante tres segundos (en el centro del panel) el mensaje "iPor haber perjudicado a un compañero has perdido 3 puntos!'. La puntuación acumulada disminuía tres puntos y, en tanto se ejecutaba la resta, el contador sobresalía debido a que se presentaba con una luz intermitente.

En relación con el grupo experimental CPNP, si el participante oprimía el botón 'Prefiero continuar con mi tarea' se presentaba durante tres segundos (en el centro del panel) el mensaje "iPor no perjudicar a un compañero has obtenido 3 puntos!'. La puntuación acumulada incrementaba tres puntos y, en tanto se ejecutaba la sumatoria, el contador sobresalía debido a que se presentaba con una luz intermitente.

Con respecto al grupo experimental CNNP, si el participante oprimía el botón 'Prefiero continuar con mi tarea' se presentaba durante tres segundos (en el centro del panel) el mensaje 'iPor no perjudicar a un compañero has perdido 3 puntos!'. La puntuación acumulada disminuía tres puntos y, en tanto se ejecutaba la resta, el contador sobresalía debido a que se presentaba con una luz intermitente. 
Y, finalmente, en cuanto al grupo control SC, si el participante perjudicaba o no a uno de los compañeros virtuales se omitía la presentación de consecuencias tanto positivas como negativas.

El procedimiento descrito, fue idéntico para los cinco grupos. En cuanto los participantes acumulaban sesenta puntos concluía la tarea experimental e inmediatamente aparecía un recuadro, en el cual, se agradecía la participación.

\section{Resultados}

El objetivo de la presente investigación, tuvo como objetivo evaluar los efectos de dos tipos de consecuencias (positivas y negativas) sobre la elección entre perjudicar/no perjudicar a un compañero en una tarea aritmética. Con el fin de evaluar este efecto se realizó un análisis individual y grupal: a) Porcentaje de perjuicio, es decir, número de veces, en las cuales, el participante tuvo oportunidad de perjudicar a uno de los compañeros virtuales y, evidentemente, eligió hacerlo; b) Porcentaje de elección perjudicar respecto de los compañeros virtuales, lo cual conlleva al número de veces, en las cuales, se eligió perjudicar y, a su vez, la frecuencia de esta elección respecto a los compañeros virtuales; c) Porcentaje de operaciones aritméticas básicas en la tarea propia, lo cual indica, el número de operaciones solucionadas referentes a la tarea propia y, a su vez, la cantidad de operaciones realizadas correctamente; d) Porcentaje de operaciones aritméticas correctas dirigidas a perjudicar, indicando, el número de operaciones realizadas con la pretensión de perjudicar $y$, a su vez, la cantidad de operaciones solucionadas correctamente; e) Porcentaje del tipo de operación aritmética realizada en referencia a la tarea propia, lo que refiere, la cantidad de operaciones realizadas durante la tarea propia y, por tanto, la cantidad de sumas, restas, multiplicaciones y divisiones; f) Porcentaje del tipo de operaciones aritméticas básicas dirigidas a perjudicar, enunciando, la cantidad de operaciones, según las cuales, el participante solucionó en aras de perjudicar y, por consiguiente, la cantidad de sumas, restas, multiplicaciones y divisiones.

En la Figura 3 se observa que el grupo experimental CNNP presenta el porcentaje superior de perjuicio $(82.78 \%)$, mientras que el grupo CPP presenta el porcentaje más bajo (2.58 $\%)$. En cuanto a los otros tres grupos, se identifica un porcentaje de perjuicio inferior al 10\%.

\section{Figura 3}

Porcentaje de perjuicio identificado en los cinco

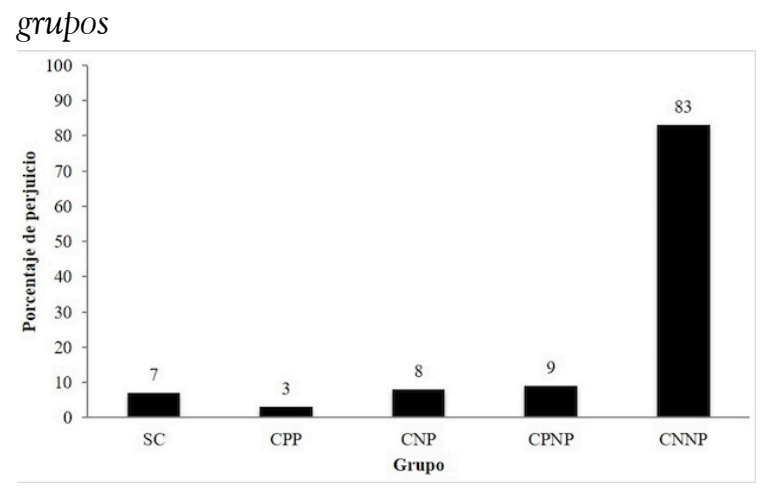

En la Figura 4 se muestran los porcentajes de perjuicio de manera individual. En cuanto al grupo SC, los participantes 1 y 6 presentaron conductas perniciosas (20\% y $26 \%$ respectivamente), mientras que los participantes 2, 5 y 7 eligieron no perjudicar. En el grupo CPP, sólo los participantes 6 y 7 eligieron perjudicar; el primero de éstos presentó el porcentaje más alto $(33 \%)$. Por otra parte, en el grupo CNP, únicamente el participante 3 eligió no perjudicar; el participante 6 perjudicó con mayor frecuencia (17\%). En relación con el grupo CPNP, sólo los participantes 1 y 7 eligieron perjudicar; el último de éstos presentó mayor porcentaje pernicioso (33 \%). Y, finalmente, en el grupo CNNP, sin excepción, los participantes eligieron perjudicar, observándose porcentajes superiores al 70\%. 
Figura 4

Porcentaje de perjuicio individual

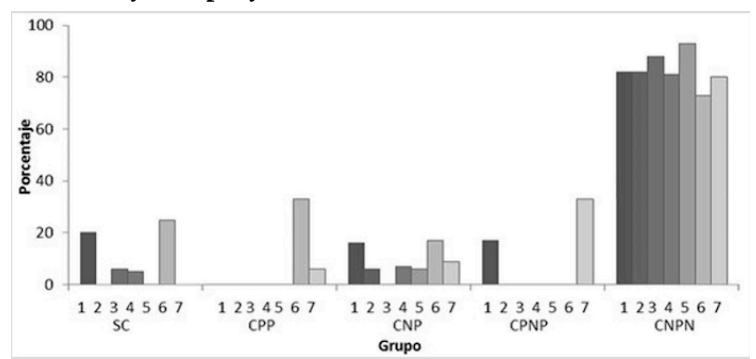

Adicionalmente, se realizó un análisis de varianza OneWayAnova, encontrando resultados significativos $F(4,30)=79.033 \mathrm{p}<0.05$ y, a su vez, la prueba Post Hoc (HSD de Tukey) mostró que las diferencias se hallan en el grupo CNNP respecto a los otros grupos. Sin embargo, no se encontraron diferencias significativas entre los grupos SC, CPP, CNP y CPNP. Asimismo, otro dato relevante, muestra que los participantes eligieron perjudicar al compañero con la puntuación superior, seguido por el compañero con una puntuación próxima o idéntica y, en menor medida, al compañero con una puntuación inferior.

Igualmente, se analizaron los datos relativos al porcentaje de operaciones aritméticas básicas solucionadas correctamente tanto en la tarea propia como en la tarea ajena, hallándose -en ambas tareas- que el porcentaje de operaciones correctas supera el 70\%. No obstante, los porcentajes superiores se identifican en la solución de operaciones referentes a la tarea ajena -a excepción del grupo CNNP.

\section{Figura 5}

Porcentaje de operaciones aritméticas solucionadas correctamente tanto en la tarea propia como en la ajena

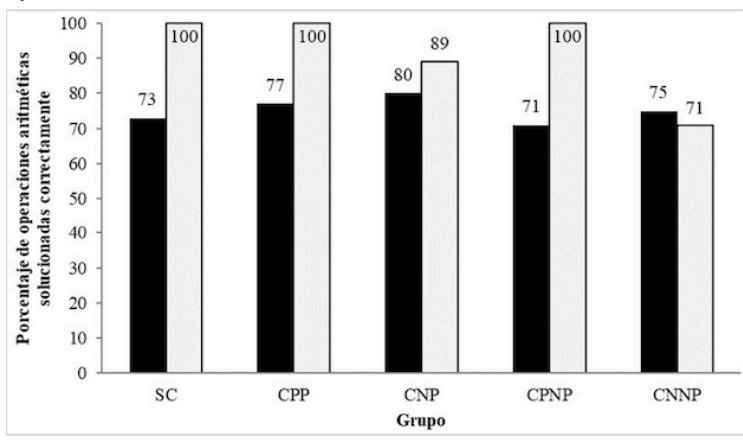

Es importante recordar que las operaciones aritméticas básicas proporcionaban puntos diferencialmente, es decir, las sumas y restas proporcionaban 1 punto, las multiplicaciones 3 puntos y las divisiones 5 puntos. Posteriormente, se graficó el tipo de operación seleccionada tanto en la tarea propia como en la ajena (ver Figuras 6 y 7). En la Figura 6 se observa que en su mayoría se realizaron multiplicaciones, se identifican porcentajes superiores al $45 \%$. Por otro lado, en la tarea ajena, se observan porcentajes similares en relación con la solución de multiplicaciones y divisiones (30\% y $35 \%$ respectivamente).

\section{Figura 6}

Tipo de operación aritmética solucionada en la tarea propia

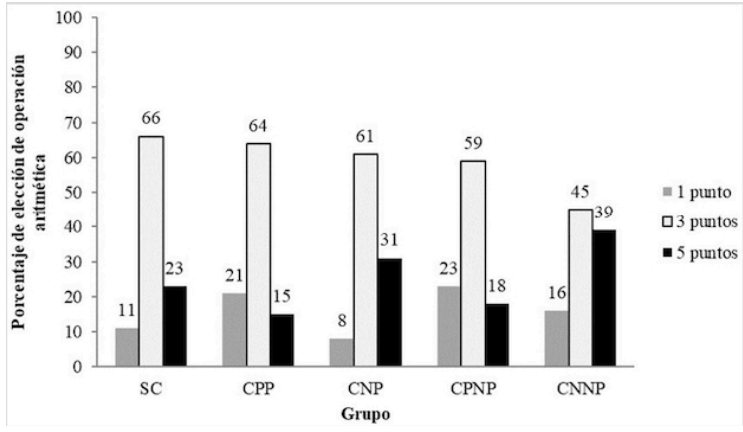




\section{Figura 7}

Tipo de operación aritmética solucionada en la tarea ajena en términos porcentuales

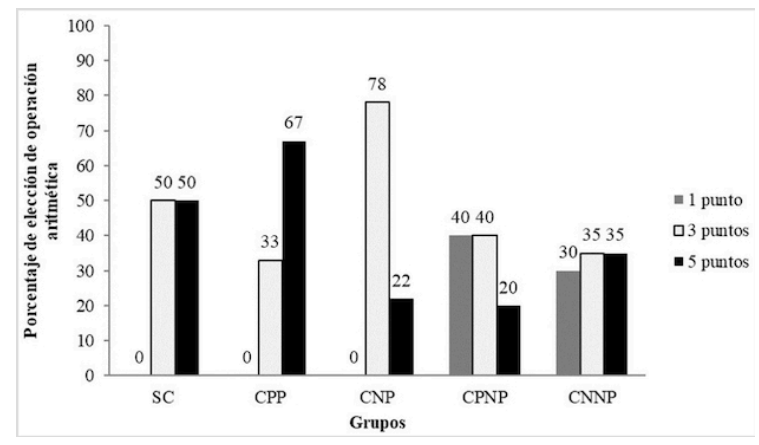

\section{Discusión}

La presente investigación tuvo como objetivo evaluar los efectos de dos tipos de consecuencias -positivas y negativas- sobre la elección entre perjudicar/no perjudicar a un compañero en una tarea aritmética y, considerando la suposición explicitada en las investigaciones sobre comportamiento solidario (Carpio et al., 2008a, 2008b). Es decir, caracterizar a las consecuencias situacionales como un factor crítico en tanto modulan aquellas interacciones se discuten los efectos de éstas en los siguientes términos.

Los participantes de los grupos SC, CPP, $\mathrm{CNP}$ y CPNP, muestran un porcentaje promedio de perjuicio similar; únicamente en el grupo experimental CNNP en el cual se encuentran diferencias significativas; estos datos coinciden con los reportados por Cantoran (2007) en cuanto a que las consecuencias negativas promueven que un individuo ayude o perjudique. El hecho de que solamente en el grupo CNNP se observe un mayor porcentaje de comportamiento pernicioso, indica que los participantes eligen continuar con la tarea propia en la medida en que no se afecte el campo contingencial de los mismos, es decir, que no se les perjudique. Por tanto, es plausible afirmar que en una situación, en la cual, sólo es posible satisfacer el criterio prescrito si se perjudica a otro individuo, la probabilidad de que se despliegue comportamiento pernicioso es muy alta y, evidentemente, aumenta si se identifica una afectación negativa respecto del campo contingencial propio. Esta afirmación converge con los datos obtenidos por Milgram (2005) durante la década de los sesenta y las réplicas realizadas por Burger (2009) puesto que se observó que la mayoría de los participantes cometen actos perjudiciales como medio para actuar de acuerdo con su criterio personal. No obstante, estas investigaciones carecen de un anclaje multifactorial, es decir, de campo.

En el análisis realizado, los miembros de los grupos CNP y CPNP, se caracterizaron por no perjudicar a un compañero. Estos resultados en ambos grupos son susceptibles de vincularse con la conceptualización skinneriana respecto de la moral debido a que en el primero de éstos se castiga la elección de perjudicar a los compañeros mientras que en el segundo se refuerza la elección contraria. Sin embargo, esta concepción es limitada en tanto se ampara dentro de un esquema causal, ignorando otros factores participantes en tales interacciones, como lo son los factores históricos que resultan de formar parte de un grupo social (Chiesa, 2003), cuya recuperación permite entender lo sucedido con estos y otros grupos de participantes. Por ejemplo, en el grupo CPP hipotéticamente, se esperaría obtener un porcentaje alto de comportamiento pernicioso similar al del grupo CNNP, empero, los datos indican lo opuesto. Un factor posiblemente relacionado con lo observado en referencia a los participantes del grupo CPP porcentajes bajos de conductas perjudiciales- es la historia ontogenética individual, atendiendo a los postulados formulados por Kantor \& Smith (1975) y Ribes y López (1985). Luego entonces, este factor sugiere que la historia individual concursó en las interacciones de los participantes durante la tarea experimental, a pesar de que en el grupo CPP se presentaron consecuencias positivas por perjudicar, los participantes eligieron no perjudicar. Asimismo, es factible suponer que estos participantes valoran desfavorablemente las contingencias perniciosas de acuerdo con las prácticas propias del grupo social al que pertenecen (Ribes et al. 2008). En otras palabras, la valoración 
compartida respecto de los actos perjudiciales determina la ocurrencia de contingencias perniciosas. De esta manera, los participantes del grupo experimental CPP se ajustaron de acuerdo con criterios valorativos particulares, los cuales, están prescritos por el grupo social de referencia.

Por otro lado, en relación con las características del desempeño de los participantes tanto en la tarea propia como en la tarea ajena, se puede observar que en los distintos grupos (a excepción del grupo CNNP) se presentó mayor efectividad en las operaciones aritméticas dirigidas a perjudicar (tarea ajena). No obstante, tiene que considerarse que en estos grupos la cantidad de operaciones realizadas durante la tarea propia, fue mayor en comparación con las operaciones realizadas en la tarea ajena. Esto sugiere que hubo mayor probabilidad de equivocaciones durante la realización de la tarea propia. En cuanto al grupo CNNP, la efectividad en la tarea propia y en la tarea ajena fue similar. Tomando en cuenta esta sutil diferencia, se robustecería la afirmación sostenida por Cantoran (2007) acerca del desempeño efectivo asimétrico en la tarea ajena respecto del desempeño efectivo de la propia, es decir, los participantes procuraron realizar de manera efectiva las operaciones aritméticas dirigidas a cumplir el criterio propio, soslayando la efectividad relativa a la tarea ajena. Dicho de otra manera, las operaciones aritméticas dirigidas a perjudicar, solamente se realizaron para procurar una afectación negativa del campo contingencial ajeno.

En cuanto al tipo de operación realizada en la tarea propia, se observó la solución de diversas operaciones. Sin embargo, las multiplicaciones se tornaron hegemónicas. En relación a los tipos de operaciones aritméticas dirigidas a perjudicar, con excepción del grupo CNNP, se identificaron elecciones asimétricas. Por tanto, se constató la inexistencia de un patrón referente a la elección de un tipo particular de operación dirigida a perjudicar. Lo anterior indica que la dificultad de las tareas no es un factor relevante en torno a la elección entre perjudicar/no perjudicar. En el grupo CNNP, se identificó un porcentaje similar en lo concerniente al tipo de operación realizada, esto sugiere que en una situación, en la cual se dispone a perjudicar con frecuencia, en la forma de hacerlo, no importa el tipo de operación. Un dato relevante atañe al participante perjudicado, es decir, en los cinco grupos el porcentaje más alto de comportamiento pernicioso, se concentró en el compañero con una puntuación superior.

Recapitulando los principales hallazgos de la presente investigación,se destaca que el porcentaje de comportamiento pernicioso es muy bajo en tanto no se presentan consecuencias situacionales negativas; a la par, que el compañero virtual con una mayor puntuación es susceptible de las contingencias dirigidas a perjudicar; adicionalmente, el tipo de operaciones realizadas en la tarea propia "poseen" una dificultad moderada (multiplicaciones); y, por último, es necesario subrayar que el comportamiento pernicioso no está determinado exclusivamente por factores biológicos y, por tanto, no es una cualidad inherente a los individuos; es susceptible de modular y probabilizar por factores tales como las consecuencias situacionales.

En breve, diversos factores participan en la estructuración del comportamiento pernicioso. Se ha evaluado el papel que desempeñan las consecuencias situacionales, sin embargo, teniendo presente los datos relativos al grupo CPP se torna relevante evaluar -en situaciones controladas- la participación de factores tales como las interacciones lingüísticas previas, lo cual quiere decir, que la historia referencial individual que permita tener un cuadro más amplio de su papel en situaciones en las que se colabora y perjudica (Pacheco, 2010). También resulta interesante realizar un análisis de grano fino, sobre la forma en la cual el perjuicio que otro ejerce en la tarea propia auspicia o modula el perjuicio que uno pudiese desplegar sobre la labor de otros. Esto permitiría dar cuenta de cómo se incorporan los episodios de perjuicio sufridos, presenciados o ejercidos en la historia de los individuos, a nivel lingüístico y situacional, así como sus posibles efectos colaterales. Con estos y otros análisis, será posible dar cuenta de fenómenos sociales manifestados a nivel individual, por ejemplo, el 'Bullying' o el 'Mobbing' 
que tanto afectan la vida personal, escolar, profesional y la propia salud en las sociedades occidentales.

\section{Agradecimientos}

Este trabajo fue posible gracias a los financiamientos de la DGAPA de la U.N.A.M. a través del programa PAPIME (proyecto PE307216), PAPIIT (proyecto IN307013) y PAPCA 36-2012 de la FES Iztacala.

\section{Referencias}

Burger, J. (2009). Replicating Milgram. Would people still obey today?. American Psychologist, 64(1), 1-11. https://doi.org/10. 1037/a0010932

Cantoran, E. (2007). Comportamiento solidario: Análisis experimental del tipo de consecuencias. (Tesis de licenciatura inédita). UNAM, FES: Iztacala.

Carpio, C., Silva, H., Pacheco-Lechón, L., Cantoran, E., Arroyo, R., ... Pacheco, V. (2008a). Efectos de las consecuencias positivas y negativas sobre la conducta altruista. Universitas Psychologica, 7(1), 97-107. Recuperado de http://revistas.javeriana.edu.co/index.p hp/revPsycho/article/view/202/213

Carpio, C., Silva, H., Reyes, A., Pacheco-Lechón, L., Morales, G., ... Pacheco, V. (2008b). Factores lingüísticos y consecuencias situacionales en la elección de estudiantes universitarios entre colaborar y no colaborar en tarea académicas: un análisis experimental. Acta Colombiana de Psicología, 11(2), 114-126. Recuperado de http://www.scielo.org.co/scielo.php?scri $\mathrm{pt}=$ sci_arttext\&pid $=$ S0123-91552008000 200011

Chiesa, M. (2003). Sobre la meta-ética, normativa y el conductismo. Revista Latinoamericana de Psicología, 35(3), 289-297. Recuperado de http://www.redaly c.org/pdf/805/80535305.pdf
Guerrero, V. (2010). Maltrato. La violencia de todos los días. ¿Cómo ves?, (143), 10-16. Recuperado el 1 de julio de 2016 de: http://www.comoves.unam.mx/assets/r evista/143/maltrato-la-violencia-de-todos-1 os-dias.pdf

Kantor, J. R. \& Smith, N. W. (1975). The science of psychology: an interbehavioral survey. Chicago: Principia Press.

Lave, J. \& Wenger, E. (1991). Situated learning: Legitimate peripheral participation. New York: Cambridge University Press. Recuperado de https://books.google.com.co/books?id= CAVIOrW3vYAC\&lpg $=$ PA11\&ots $=\mathrm{OC}$ mCvmXICn\&dq=Lave $\% 2$ C $\% 20 \mathrm{~J} . \% 20 \% 2$ 6\%20Wenger\%2C\%20E.\%20(1991).\%20S ituated\%20learning\%3A\%20Legitimate\% 20peripheral\%20participation.\%20New\%2 OYork\%3A\%20Cambridge\%20University $\% 20$ Press.\&lr\&hl $=$ es\&pg $=$ PA5 $\#_{\mathrm{v}}=$ onep age $\& q \& f=$ false

Lima, T. D. F. \& Souza, M. A. (2015). O impacto do Mobbing sobre o estrese no trabalho. Estudos e Pesquisas em Psicologia, 15(2), 608-630. https://doi.org/10.12957/epp.201 5.17661

Milgram, S. (2005). Los peligros de la obediencia. Polis. Revista de la Universidad Bolivariana,4(11). Recuperado el 1 de julio de 2016 de: http://www.redalyc.org/articul o.oa? id $=30541124$

Pacheco, L. (2010). Elección colaborar/no colaborar en estudiantes universitarios. Efectos de la historia con el compañero. (Tesis de licenciatura inédita). UNAM, FES: Iztacala. Recuperado de http://132.248.9.195/ptb2010/noviembr e/0664456/Index.html

Rocha-Sánchez , R. y Díaz-Loving, R. (2005). Cultura de género: la brecha ideológica entre hombres y mujeres. Anales de Psicología. 21(1), 42-49. Recuperado de https://digitum.um.es/xmlui/bitstream/1 0201/8056/1/Cultura\%20de\%20genero.\%2 OLa\%20brecha\%20ideologica\%20entre\%2 Ohombres\%20y\%20mujeres.pdf 
Ribes, E. \& López, F. (1985). Teoría de la conducta. Un análisis de campo y paramétrico. México: Trillas.

Ribes-Iñesta, E., Rangel, N. E., \& LópezValadez, F. (2008). Análisis teórico de las dimensiones funcionales del comportamiento social. Revista Mexicana de Psicología, 25(1), 45-57. Recuperado de http://colpos.redalyc.org/articulo.oa?id $=243016300003$

Rogoff, B. (1993). Aprendices del pensamiento. El desarrollo cognitivo en el contexto social. Barcelona: Paidós.

Van der Werf, C. (2014). The effects of Bullying on academic achievement. Desarrollo y Sociedad, (74), 275-308. Recuperado de https://revistas.uniandes.edu.co/doi/abs/ $10.13043 /$ dys. 74.6

\section{Notas}

* Artículo de investigación. 